\title{
INVESTIGACIÓN/RESEARCH
}

\section{MULTICULTURALIDAD, INTERCULTURALIDAD Y DESARROLLO PERSONAL EN EL EEES}

María D. De-Juan-Vigaray ${ }^{1}$ : Universidad de Alicante (España) mayo@ua.es

Ma Concepción Parra Meroño: Universidad Católica de Murcia (España) mcparra@ucam.edu

Miguel Ángel Beltrán Bueno: Universidad Católica de Murcia (España) mabeltran@ucam.edu

\section{RESUMEN:}

En el EEES conviven a diario en las aulas universitarias estudiantes de diferentes nacionalidades y culturas. Esta situación, propiciada en gran parte por el programa ERASMUS, dota a la universidad actual de un ámbito multicultural sin precedentes. Sin embargo, si queremos dar un paso más allá de la multiculturalidad será necesario una mayor involucración de docentes y discentes.

En este contexto, nuestro objetivo es lograr la interculturalidad en las aulas actuales. Para ello hemos considerado el trabajo cooperativo en pequeños grupos como potenciador de dicho concepto. Adoptando un enfoque metodológico cualitativo, se preguntó a los estudiantes, mediante entrevistas personales su percepción del trabajo realizado.

Los alumnos destacan las ventajas del trabajo cooperativo, con efectos positivos sobre el desarrollo personal de cada uno de los participantes con el consiguiente enriquecimiento tanto en la forma de trabajo como en el conocimiento más directo de distintos modos de vida y distintas formas de pensar y de afrontar los problemas.

PALABRAS CLAVE: Multiculturalidad-Interculturalidad-Desarrollo Personal-EEESAprendizaje Cooperativo

\footnotetext{
${ }^{1}$ María D. De-Juan-Vigaray: Universidad de Alicante/ Departamento de Marketing. Facultad de Ciencias Económicas y Empresariales/España. Campus de San Vicente del Raspeig, s/n. Alicante.

Correo institucional: mayo@ua.es
} 


\section{Multiculturalism, Cross-cultural, Personal development in the EHEA}

\section{ABSTRACT:}

In the EHEA students from different nations and cultures live and learn side-by-side in university lecture halls every day. This situation, to a great extent fostered by the ERASMUS programme, creates an unprecedented multicultural environment in contemporary universities. However, if we want to take a step beyond multiculturalism, greater involvement is needed on the part of teachers and students. With this in mind, our aim is to achieve cross-cultural environment in the classrooms of today. In order to do this, we have considered teamwork in small groups to encourage this concept. Adopting a qualitative methodological focus, in individual interviews students were asked their view of the work carried out.

The students highlighted the advantages of working in a team, it having positive effects upon the personal development of each of the participants and proving to be an enriching experience, both in the way they worked, as well as in the more direct knowledge acquired on different ways of life and ways of thinking and dealing with problems.

KEY WORDS: Multicultural - cross-cultural - Personal development - EHEA Cooperative learning

\section{INTRODUCCIÓN}

En el mundo globalizado en el que vivimos, las universidades han ampliado su público objetivo desde lo nacional hacia lo internacional. Nuestras aulas están hoy en día compuestas por alumnos de diferentes nacionalidades y culturas. Esto se debe principalmente al programa Erasmus y a la implantación del denominado proceso de Bolonia que propugna el Espacio Europeo de Educación Superior (EEES). Este sistema de enseñanza aprendizaje europeo, supone el reconocimiento de los estudios de Grado en cualquier país de los integrantes de dicho Espacio de Educación. Así, además, se potencia el intercambio cultural, favorecido por el hecho de que los estudiantes pueden cursar un cuatrimestre $o$ un curso completo en otro país diferente al suyo de origen, de modo que las asignaturas que superen en ese otro Estado, les serán reconocidas en su universidad de origen. Esta situación supone un innegable intercambio cultural entre los distintos pueblos, que se da sobre todo entre los jóvenes.

Sin embargo, nuestra experiencia como docentes universitarios, nos ha permitido observar, cómo los estudiantes extranjeros no se integran con plenitud en nuestras aulas, formando entre sí sus propios grupos de trabajo incluso trabajando a nivel individual. 
En este contexto, nos planteamos averiguar si la realidad en el aula es multicultural o más bien intercultural, con las diferencias que ello supondría para los estudiantes y profesorado. Para ello, a continuación se plantean los objetivos del estudio. En siguiente lugar se presenta la revisión de la literatura pertinente que nos permitirá contar con un fundamento teórico, para plantear la metodología del estudio empírico, presentar los resultados de las entrevistas realizadas y elaborar las conclusiones de la investigación.

En este epígrafe se abordan y explican los conceptos clave de la investigación. En primer lugar habrá que diferenciar entre los términos "multiculturalidad" e "interculturalidad", que si bien son parecidos e incluso se utilizan de forma indistinta en ocasiones, tienen significados y matices importantes que los diferencian y que en el contexto que los aplicamos tienen además grandes repercusiones en la formación integral de los estudiantes y por ende, en su desarrollo personal. A continuación, se explica la labor de la educación como instrumento imprescindible para valorar y respetar la diversidad cultural y favorecer la comunicación y competencia interculturales. Así, centraremos la revisión teórica en dos aspectos clave, el programa Erasmus y su repercusión y el aprendizaje colaborativo como motor de la educación intercultural.

\subsection{Multiculturalidad vs. interculturalidad}

Podríamos comenzar con la siguiente frase:

Vivimos en una sociedad multicultural. España es un conjunto de pueblos de diversas culturas y lenguas tradicionales. A nuestro mosaico pluricultural se siguen incorporando miles de personas con nuevas lenguas y culturas. Estamos además aprendiendo la hermosa y difícil construcción de una Europa unida, compuesta también por numerosos pueblos (Muñoz Sedano, 1997).

Más de casi dos décadas después, seguimos involucrados en ese proceso de integración cultural y de construcción. Eso sí, cada día más conscientes de su importancia.

Señalaremos las diferencias entre Multiculturalidad e interculturalidad. Nos referimos con el término multiculturalidad al conjunto, debidamente articulado, de elementos relacionados con la forma de pensar, sentir y actuar, ligada a creencias básicas y generales que dan a los diversos grupos culturales un grado más o menos elevado de cohesión. Este concepto no implica un enriquecimiento entre culturas.

Por otro lado, el concepto interculturalidad sí que implica la comunicación comprensiva entre las distintas culturas que conviven en un mismo espacio, dándose un enriquecimiento mutuo, y un reconocimiento y valoración entre las distintas culturas, en un marco de igualdad.

De este modo y atendiendo a las definiciones que apuntan diversos autores como Quintana (1992), Jordan (1996) y Del Arco (1998) el término multiculturalidad hace referencia única y exclusivamente a la yuxtaposición de las distintas culturas existentes en un mismo espacio físico, pero sin que implique que haya un enriquecimiento, es decir, sin que haya intercambio entre ellas. Sin embargo, y siguiendo a Buendía (1992), Quintana (1992), Jordan (1996) y Del Arco (1998), el término interculturalidad, implica una comunicación comprensiva entre las distintas culturas que conviven en un mismo espacio, siendo a través de éstas donde se produce el enriquecimiento mutuo y por consiguiente, el reconocimiento y la 
valoración (tanto intrínseca como extrínseca) de cada una de las culturas en un marco de igualdad.

Cultura es en definitiva aprendizaje. Es todo lo que se aprende y se transmite socialmente, y nuestra sociedad es una sociedad multicultural.

\subsection{Educación: aprendizaje colaborativo}

La educación es uno de los principales instrumentos para valorar y respetar la diversidad cultural y favorecer la comunicación y competencia interculturales. Vamos a hacer, por tanto, hincapié en estos dos aspectos. En cómo enfocar la educación en un entorno multicultural para conseguir las mayores ventajas en cuanto al desarrollo personal de los estudiantes.

La definición de Amani (2004) explicita precisamente la idea que combina los conceptos de educación y de intercultural: "La Educación Intercultural es una educación que promueve el encuentro entre personas en igualdad, contrarrestando cualquier forma de exclusión, fomentando valores y actitudes necesarias para llevar a cabo experiencias interculturales y desarrollando transformaciones estructurales necesarias para poder poner en práctica estos valores".

Es por tanto una realidad compleja y variada, que debe buscar la implementación del pluralismo y la diversidad en todas las propuestas educativas, partiendo del respeto y la tolerancia, y convirtiendo el choque cultural en algo positivo y enriquecedor. Este tipo de educación llevará a convertir nuestra sociedad en una sociedad intercultural, y no sólo multicultural, con todo lo que ello implica.

\subsection{Aplicación al Plan Bolonia}

En cuanto a las aulas universitarias de hoy, es preciso indicar que el trabajo colaborativo entre los alumnos de diferentes nacionalidades y culturas se ha visto incrementado desde la implementación del Plan Bolonia. No sólo por el incremento de las cifras en cuanto a distintas culturas en las aulas, como a continuación veremos, sino también por el cambio de estilo de metodología. El trabajo en grupo está siendo aplicado por muchos docentes.

No obstante, ya se venía dando años atrás este trabajo colaborativo debido a las ventajas que puede tener a la hora de trabajar con estudiantes extranjeros, por ejemplo Erasmus, dado que normalmente facilita la integración de los mismos.

Un trabajo colaborativo puede verse favorecido por las nuevas tecnologías. Los estudiantes extranjeros pueden ver su tarea facilitada con las herramientas que proporciona Internet. En cuanto a los docentes, son esenciales para llevar a cabo una educación intercultural, por lo que tendrán que estar concienciados en hacer efectivo este proceso de integración y enriquecimiento común. La aplicación de programas formativos para los profesores podría ayudar en esta tarea, orientándoles en determinadas situaciones y llevándoles a la reflexión y la sensibilidad hacia la diversidad.

El objetivo principal sería acercar de forma significativa para toda la comunidad educativa la teoría de la educación intercultural a las prácticas cotidianas. Así, EEES pone en el centro del proceso de enseñanza aprendizaje al alumno y pretende que éste adquiera conocimientos, pero sobre todo que alcance competencias. Por tanto, 
los sistemas que se desarrollen deben permitir a los estudiantes un avance progresivo en su desarrollo personal en varias dimensiones; incrementar su saber e ir descubriendo y comprendiendo la variedad y complejidad del mundo que los rodea; despertar la curiosidad intelectual; estimular el sentido crítico; y adquirir una mayor y progresiva autonomía (Parra y Peña, 2012).

Así, en el contexto multicultural en el que se encuentran las universidades, el diseño de enfoques docentes más plurales capaces de incrementar la participación de los estudiantes en su proceso de aprendizaje, pueden cumplir un doble objetivo, la integración de estudiantes de diferentes nacionalidades y culturas y la implicación de los alumnos en su proceso de aprendizaje. Entre dichos enfoques De Miguel Díaz et al. (2006a) destacan el "aprendizaje cooperativo", cuyo propósito principal es que los estudiantes aprendan de modo activo aprovechando las sinergias que se produzcan entre ellos.

Este aprendizaje cooperativo se basa en grupos de trabajo de tamaño pequeño, en los que cada miembro del equipo es responsable de un resultado, el cual es inalcanzable a menos que todos ellos trabajen juntos (Trujillo Sáez, 2002). Además, prioriza la colaboración y la cooperación frente a la competencia y posibilita el aprendizaje basado en competencias (De Miguel Díaz, 2006b).

\subsection{Estudiantes extranjeros en España}

Desde que se inició el programa Erasmus en 1987 cientos de miles de jóvenes europeos cursan parte de sus estudios universitarios en otros países que forman parte de este sistema de intercambio. Las principales ventajas son conocidas por todos los universitarios, se perfecciona un idioma extranjero a la par que se cursan un número de créditos (asignaturas) que una vez superadas serán automáticamente convalidadas en su universidad de origen.

Como se puede apreciar en la Figura 1, las cifras de estudiantes extranjeros matriculados en el Sistema Universitario Español para los cursos 2010/11 y 2011/12fueronmuy elevadas. Se observa que en España, más del 4 por ciento de los estudiantes universitarios son extranjeros, y en lo que se refiere a los cursos de Máster llega a superar el 17 por ciento.

\begin{tabular}{|c|c|c|c|c|c|c|c|c|}
\hline & \multicolumn{4}{|c|}{ Curso 2011-12 } & \multicolumn{4}{|c|}{ Curso 2010-11 } \\
\hline & \multirow{2}{*}{ Total } & \multicolumn{3}{|c|}{ Extranjeros } & \multirow{2}{*}{ Total } & \multicolumn{3}{|c|}{ Extranjeros } \\
\hline & & Total & \%Total & UE-27 & & Total & $\begin{array}{l}\% \\
\text { Total }\end{array}$ & UE-27 \\
\hline Total & 1.582 .714 & 73.076 & $4,6 \%$ & 24.929 & 1.529 .862 & 63.879 & $4,2 \%$ & 22.379 \\
\hline $\begin{array}{l}\text { Estudiantes } \\
\text { de Grado }\end{array}$ & 824.741 & 34.236 & $4,2 \%$ & 12.783 & 537.090 & 20.206 & $3,8 \%$ & 7.522 \\
\hline
\end{tabular}


Estudiantes

de 1 er y $\mathbf{2}^{\circ}$

Ciclo

$644.912 \quad 18.977$

$2,9 \% \quad 7.732$

$887.928 \quad 25.354$

$2,9 \%$

10.344

\section{Estudiantes}

de Máster

113.061

19.863

$17,6 \%$

4.414

$104.844 \quad 18.319 \quad 17,5 \%$

4.513

Oficial

Figura 1: Estudiantes extranjeros matriculados en el Sistema Universitario Español Fuente: Ministerio de Educación, Cultura y Deporte

Por otro lado, como se observa en la Figura 2, tanto las entradas como las salidas de estudiantes Erasmus en España han ido incrementándose en la última década, incluidos los años de la crisis económica para los que hay datos.

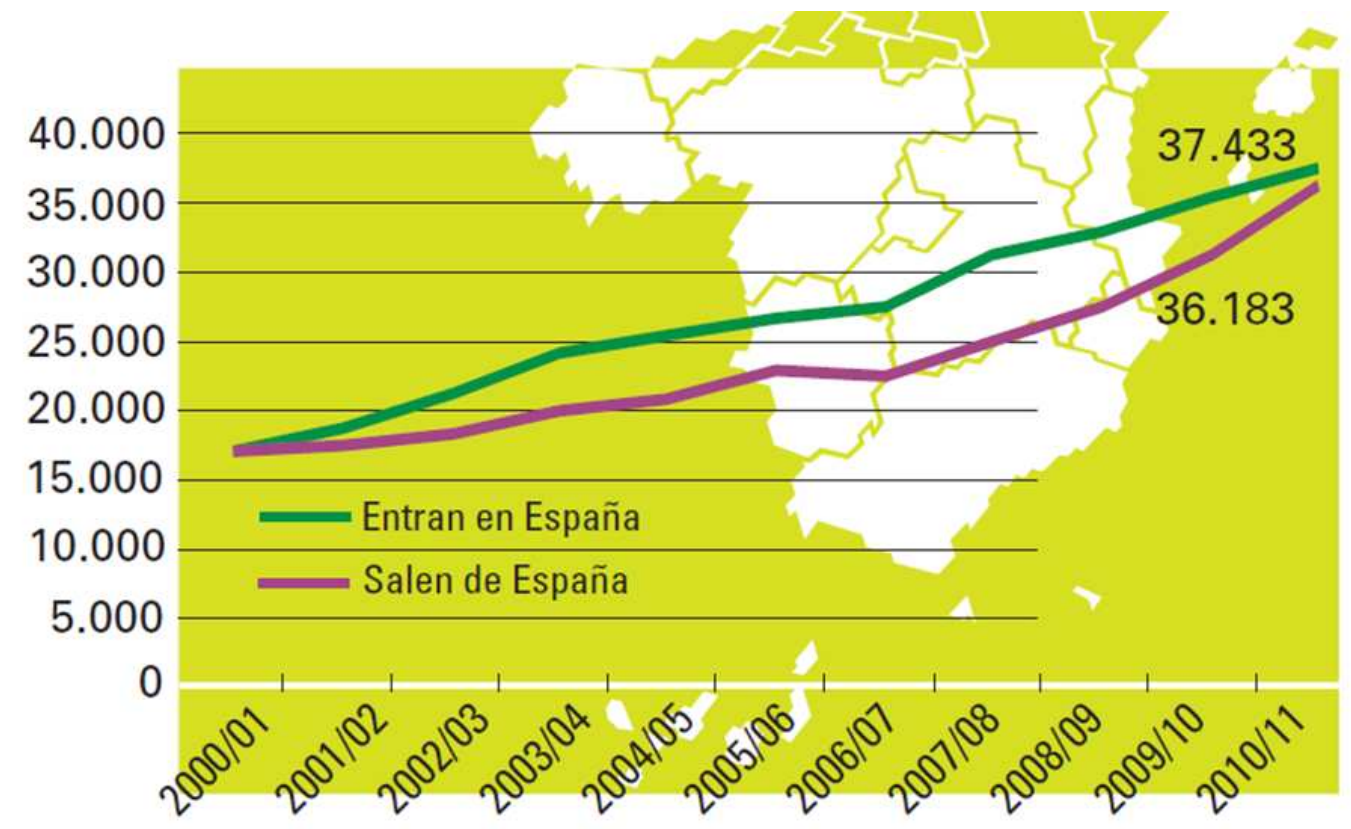

Figura 2. España y los estudiantes Erasmus Fuente: INE y Comisión Europea (2012/13)

A las cifras anteriores hay que añadir, que España es el principal receptor de estudiantes Erasmus así como el principal emisor de este tipo de estudiantes (véase la Figura 3). 


\begin{tabular}{|c|c|}
\hline \multicolumn{2}{|c|}{ MOVILIDAD ESTUDIANTIL. ERASMUS 2010/2011 } \\
\hline 5 PRINCIPALES PAISES EMISORES & 5PRINCIPALES PAISES RECEPTORES \\
\hline España & España \\
Francia & Francia \\
Alemania & Alemania \\
Italia & Reino unido \\
Polonia & Italia \\
\hline
\end{tabular}

Figura 3: Movilidad Estudiantil. Erasmus curso 2010/11

Fuente: Comisión Europea

Estos datos corroboran, la necesidad de aplicar un sistema de educación y desarrollo personal intercultural, de aprendizaje y enriquecimiento mutuo entre los estudiantes de diferentes nacionalidades. Para ello, contamos con la opinión de algunos estudiantes respecto al trabajo en grupo con estudiantes extranjeros.

\section{OBJETIVOS}

El objetivo principal de este trabajo es doble: Por un lado, conocer si en las aulas -en las que a priori hay multiculturalidad-, hay además interculturalidad $y$, por otro, comprobar si ésta contribuye al desarrollo personal de los estudiantes. Finalmente, ambos objetivos nos permitirán determinar las ventajas y desventajas que este entorno pueda implicar en el aula y en los futuros profesionales.

\section{METODOLOGÍA}

Con el propósito de que el aula sea intercultural y no solo multicultural se utilizó una metodología activa de enseñanza aprendizaje para las prácticas de las asignaturas objeto de estudio. Se decidió utilizar el trabajo cooperativo, de modo que los estudiantes de diferentes nacionalidades tuvieran la oportunidad de trabajar juntos. Para ello se formaron grupos reducidos de entre tres y cinco estudiantes cada uno.

Una vez finalizado el curso, para extraer conclusiones hemos realizado entre los alumnos un estudio con un enfoque metodológico cualitativo. Para ello, hemos realizado entrevistas a estudiantes que durante el curso 2013/14 han estado inmersos en ambientes multiculturales o interculturales. Se han tenido en cuenta las opiniones de diversos tipos de perfiles de estudiantes:

1. Las opiniones de los estudiantes españoles en su país de origen (en España)en relación con sus compañeros de otros países,

2. Las opiniones de los propios estudiantes españoles que han partido a otros países como estudiantes Erasmus 
3. Las opiniones de los estudiantes extranjeros en España.

Las entrevistas se realizaron durante el primer y segundo cuatrimestre del curso 2013/14 una vez terminadas las asignaturas correspondientes, todas ellas en el campo del marketing. Las Universidades objeto de estudio fueron una pública, Universidad de Alicante, y otra privada, la Universidad Católica de Murcia. A continuación se presentan las asignaturas escogidas y los detalles de cómo se organizaron los estudiantes para promover la interculturalidad.

1. En el $3^{\circ}$ curso del Grado en Economía, la asignatura escogida fue "Fundamentos de Marketing" 6 ECTS, que contaba con 4 horas semanales de contacto de los estudiantes en el aula. Este grupo constaba de 88 estudiantes ( 44 hombres y 44 mujeres, de los cuales 11 ( 4 mujeres y 7 hombres) eran estudiantes ERASMUS. Los estudiantes estaban divididos en 16 grupos de entre 4 y 5 miembros. En ningún grupo había más de 1 estudiante extranjero. Los estudiantes fueron asignados por la profesora totalmente al azar. De la totalidad de los grupos tres accedieron a participar en nuestra investigación (tanto los estudiantes españoles como los extranjeros) compartiendo su experiencia intercultural. Las nacionalidades de los estudiantes extranjeros participantes fueron: china, holandesa e italiana.

2. En el $4^{\circ}$ curso del Grado en Administración y Dirección de Empresas la asignatura escogida fue "Distribución Comercial" 6 ECTS, con las mismas horas de contacto de los estudiantes en clase. Este grupo constaba de 46 personas (13 varones y 33 féminas) de los cuales sólo 5 eran estudiantes extranjeros (3 varones y 2 mujeres). Siguiendo el mismo procedimiento que en el caso anterior se formaron 7 grupos en clase de los cuales dos de ellos accedieron a participar en nuestro estudio. Las nacionalidades de los estudiantes extranjeros participantes fueron francesa y rumana.

3. Se utilizó también para este estudio una asignatura de un curso de experto impartido en inglés en el marco del International Business Program, correspondiente a nivel de Máster y denominada "Retail Marketing: New Perspectives". Esta asignatura contaba con 46 estudiantes(11 hombres y 35 mujeres) y 9 grupos con estudiantes de 13 países (19 de EE.UU, 8 alemanes, 1 de Armenia, 3 holandeses, 3 irlandeses, 1 italiana, 2 lituanas, 1 eslovaco, 1 escocés, 1 español y 1 británico). De todos ellos 1 grupo accedió a participar en el estudio.

4. Un grupo de estudiantes de $3^{\circ}$ del Grado de Administración y Dirección de Empresas. Asignatura "Comportamiento del consumidor", 4,5 ECTS. Las horas de contacto de los estudiantes en el aula fueron 3 semanales. Este grupo está compuesto por 26 alumnos de los cuales 8 son extranjeros de diferentes nacionalidades. Los grupos de trabajo estaban compuestos por 3 estudiantes, y se dejo libertad para que fuesen ellos quienes eligieran a sus compañeros de grupo. De todos ellos, un total de 20 accedieron a participar en el estudio.

5. Finalmente se recogió la opinión de tres estudiantes españoles que habían estado de Erasmus durante el cuatrimestre, uno en Inglaterra cursando la asignatura Marketing Management en un grupo de unos 40 estudiantes, con un $85 \%$ de estudiantes nativos, y el resto alumnos chinos, siendo él el único estudiante español. El segundo estudiante español realizó su estancia Erasmus en Francia, en un ambiente multicultural, cursando Administración y Dirección de Empresas. El tercero realizó un curso completo de $A D E$ en Estados Unidos en un aula con estudiantes de diferentes nacionalidades.

A los estudiantes que voluntariamente accedieron a formar parte del estudio les preguntamos las siguientes cuestiones:

En relación con la asignatura $X$ : 


\section{ESTUDIANTES ERASMUS:}

1. ¿Elegiste libremente tu grupo? Si no fue así ¿Cuál fue el método de elección de tu grupo?

2. ¿Qué ha supuesto para ti trabajar con estudiantes españoles (o de otras nacionalidades diferentes a la tuya) en la clase, en general?

En relación con tu grupo de prácticas:

3. A tu parecer ¿Cuáles son las ventajas del trabajo en grupo con estudiantes extranjeros?

4. ¿Y las desventajas del trabajo en grupo con estudiantes de otros países?

5. Valoración personal de la experiencia con estudiantes españoles

6. Valoración personal de la experiencia con otros estudiantes extranjeros.

\section{ESTUDIANTES NATIVOS:}

1. ¿Elegiste libremente tu grupo? Si no fue así ¿Cuál fue el método de elección de tu grupo?

2. ¿Qué ha supuesto para ti trabajar con estudiantes extranjeros en la clase, en general?

En relación con tu grupo de prácticas:

3. A tu parecer, ¿Cuáles son las ventajas del trabajo en grupo con estudiantes extranjeros?

4. ¿Y las desventajas del trabajo en grupo en un ambiente internacional?

5. Valoración personal de la experiencia con estudiantes españoles

6. Valoración personal de la experiencia con otros estudiantes extranjeros

\section{RESULTADOS}

En este apartado se muestran las opiniones de los estudiantes sobre el trabajo colaborativo con estudiantes de otros países diferentes al suyo de origen.

Para una mayor claridad y veracidad hemos transcrito las opiniones que nos han resultado más interesantes y que recogen el sentir más común de todos los participantes en el estudio.

Tabla 1. Opiniones de los estudiantes de Economía y ADE (Universidad de Alicante)

\begin{tabular}{|c|c|}
\hline $\begin{array}{c}\text { TIPO DE } \\
\text { ESTUDIANTE }\end{array}$ & COMENTARIO \\
\hline $\begin{array}{l}\text { Español en } \\
\text { España }\end{array}$ & $\begin{array}{l}\text { "Trabajar con un compañero de otra nacionalidad tiene su parte } \\
\text { buena y su parte mala. Es interesante porque sirve para que tanto él } \\
\text { como nosotros aprendamos más sobre la cultura del otro y } \\
\text { también es divertido. Sin embargo, si el estudiante no domina } \\
\text { demasiado el idioma su trabajo en el grupo no es muy } \\
\text { intenso. Por ejemplo, nosotros le decíamos lo que tenía que hacer y } \\
\text { él lo hacía lo mejor que podía, pero nos tocaba repasar y corregir } \\
\text { bastantes cosas de su trabajo." }\end{array}$ \\
\hline Español en & "[...] el grupo trabaja más unido porque entre todos tenemos que \\
\hline
\end{tabular}




\begin{tabular}{|c|c|}
\hline $\begin{array}{c}\text { TIPO DE } \\
\text { ESTUDIANTE }\end{array}$ & COMENTARIO \\
\hline España & $\begin{array}{l}\text { ayudarle a él a que sepa lo que tiene que hacer y a que lo entienda. } \\
\text { También practicas inglés si hay algo que él no entienda en } \\
\text { nuestra lengua." }\end{array}$ \\
\hline $\begin{array}{l}\text { Español en } \\
\text { España }\end{array}$ & $\begin{array}{l}\text { "[... al estar con un compañero de otra nacionalidad los trabajos } \\
\text { en general suelen tardar más tiempo en hacerse y tú debes } \\
\text { asumir más responsabilidad [...]" }\end{array}$ \\
\hline $\begin{array}{l}\text { Español en } \\
\text { España }\end{array}$ & $\begin{array}{l}\text { "Para mí, en general, pesan más los beneficios porque soy muy } \\
\text { curioso y me llama mucho la atención saber sobre otras culturas y } \\
\text { también me gusta practicar el inglés con ellos si puedo, porque es } \\
\text { algo que también me interesa a mí y me viene bien." }\end{array}$ \\
\hline $\begin{array}{l}\text { Española en } \\
\text { España }\end{array}$ & $\begin{array}{l}\text { "[... Me ha encantado la experiencia de poder conocer otras formas } \\
\text { de trabajar teniendo compañeros extranjeros en el aula y en } \\
\text { particular en el grupo. Me ha parecido muy enriquecedor. Además } \\
\text { con el trabajo en grupo se han llegado a entablar amistades que } \\
\text { duran tras finalizar el curso de la asignatura." }\end{array}$ \\
\hline \multirow{2}{*}{$\begin{array}{l}\text { Italiano en } \\
\text { España } \\
\text { (Erasmus) }\end{array}$} & $\begin{array}{l}\text { "Es útil la experiencia de trabajar con españoles porque aprendes } \\
\text { cosas nuevas y una manera nueva de estudiar: en mi país es casi } \\
\text { todo teoría y aquí he profundizado más en la práctica. [...] Además } \\
\text { he mejorado mucho el idioma" }\end{array}$ \\
\hline & $\begin{array}{l}\text { "[...]Como problema, a veces sentía la dificultad de demostrar mis } \\
\text { conocimientos y lo que sabía hacer por la falta de manejo del } \\
\text { español, eso implicó una falta de confianza en el trabajo del grupo. } \\
\text { [...] Destacan los beneficios y ime ha encantado la experiencia!" }\end{array}$ \\
\hline $\begin{array}{l}\text { Español en } \\
\text { España }\end{array}$ & $\begin{array}{l}\text { "El trabajo en grupo con estudiantes de otra nacionalidad es, en } \\
\text { general, bastante positivo. A pesar de que a veces es dificil } \\
\text { coordinarlo porque no entienden bien lo que tienen que hacer y hay } \\
\text { que explicarlo varias veces, se implicaron mucho en los trabajos y } \\
\text { hubo un buen trabajo en equipo". }\end{array}$ \\
\hline $\begin{array}{l}\text { Rumana en } \\
\text { España } \\
\text { (Erasmus) }\end{array}$ & $\begin{array}{l}\text { "En ocasiones los estudiantes del país de acogida infravaloran al } \\
\text { estudiante internacional porque no sabe expresarse y exprimirse } \\
\text { como un nativo" }\end{array}$ \\
\hline
\end{tabular}


Tabla 2. Opiniones de los estudiantes de nivel Máster en un curso internacional impartido en inglés

\begin{tabular}{|l|l|}
\hline $\begin{array}{c}\text { Tipo de } \\
\text { estudiante }\end{array}$ & \multicolumn{1}{c|}{ Comentario } \\
\hline $\begin{array}{l}\text { Red group } \\
\text { students } \\
\text { in Spain }\end{array}$ & $\begin{array}{l}\text { "[... Then we took the photos and enjoyed some frozen yoghurt. At } \\
\text { times difficult as with } 7 \text { and different nationalities, communication } \\
\text { can beetings and the facebook group we have completed this asignment } \\
\text { with aplomb" }\end{array}$ \\
\hline
\end{tabular}

Tabla 3. Opinión de estudiantes españoles en el extranjero

\begin{tabular}{|l|l|}
\hline $\begin{array}{c}\text { Tipo de } \\
\text { estudiante }\end{array}$ & \multicolumn{1}{|c|}{ Comentario } \\
\hline $\begin{array}{l}\text { Estudiante } \\
\text { español } \\
\text { en Irlanda } \\
\text { (Erasmus) }\end{array}$ & $\begin{array}{l}\text { " [... con los chinos es esencial que uno tome el liderazgo (se sienten } \\
\text { contrario en estudiantes de países de norte de Europa, donde por lo } \\
\text { general éstos tratan de llevar la iniciativa" }\end{array}$ \\
\hline $\begin{array}{l}\text { Estudiante } \\
\text { español } \\
\text { en Francia } \\
\text { (Erasmus) }\end{array}$ & $\begin{array}{l}\text { La mayoría de casos y ejemplos se basan en hecho del país en el que te } \\
\text { encuentras y el desconocimiento del mercado y empresas hace que te } \\
\text { encuentres en desventaja }\end{array}$ \\
\hline $\begin{array}{l}\text { Estudiante } \\
\text { español } \\
\text { en USA }\end{array}$ & $\begin{array}{l}\text { Sistema de enseñanza y educación diferente. El tiempo de adaptación al } \\
\text { nuevo sistema y a los compañeros y profesores es un gran } \\
\text { inconveniente. Sin embargo, los conocimientos en profundidad de mi } \\
\text { país puede aportar cosas nuevas en clase debido a que hay muchas } \\
\text { diferencias }\end{array}$ \\
\hline
\end{tabular}

Tabla 4. Opinión de estudiantes de ADE (Universidad Católica de Murcia)

\begin{tabular}{|l|l|}
\hline $\begin{array}{c}\text { Tipo de } \\
\text { estudiante }\end{array}$ & \multicolumn{1}{c|}{ Comentario } \\
\hline $\begin{array}{l}\text { Argentina } \\
\text { en España }\end{array}$ & $\begin{array}{l}\text { "Practicar otros idiomas como el inglés,..., saber más de otras culturas y } \\
\text { países que no conocemos" } \\
\text { "Generalmente, los Erasmus se juntan entre ellos. Creo que sería bueno }\end{array}$ \\
\hline
\end{tabular}




\begin{tabular}{|c|c|}
\hline & Comentario \\
\hline & $\begin{array}{l}\text { que la Universidad realice una bienvenida, una presentación o una } \\
\text { reunión, para que sea más fácil la interacción entre los Erasmus y los } \\
\text { alumnos" }\end{array}$ \\
\hline $\begin{array}{l}\text { Francesa } \\
\text { en España } \\
\text { (Erasmus) }\end{array}$ & $\begin{array}{l}\text { "Es el momento de crear vínculos con personas de un país al que quizás } \\
\text { nunca podrías ir" } \\
\text { "Los Erasmus favorecen a los alumnos nativos a hacerse también } \\
\text { Erasmus en otro país y vivir la experiencia, con lo que tener Erasmus en } \\
\text { la misma aula puede favorecer la demanda de ir al extranjero de los } \\
\text { nativos" }\end{array}$ \\
\hline $\begin{array}{l}\text { Italiana en } \\
\text { España } \\
\text { (Erasmus) }\end{array}$ & $\begin{array}{l}\text { "Las ventajas de ser Erasmus son conocer otra cultura, abre tu mente } \\
\text { porque tienes que comprender lo que te quieren explicar y también lo } \\
\text { que pasa en ese país" } \\
\text { "Es una experiencia muy positiva y se puede aprender algo más que no } \\
\text { se encuentra en los libros" }\end{array}$ \\
\hline $\begin{array}{l}\text { Español } \\
\text { en España }\end{array}$ & $\begin{array}{l}\text { "Sentirse realizado al colaborar con el aprendizaje de estos alumnos" } \\
\text { "Muchos Erasmus ven nuestro país como fiesta y no asisten a clase para } \\
\text { así estar preparados para la noche" } \\
\text { "Pueden crearse vínculos de amistad muy fuertes que perduren en el } \\
\text { tiempo y sobrevivan a la distancia" }\end{array}$ \\
\hline aña & $\begin{array}{l}\text { "Tener amigos extranjeros ayuda a tener más interés de conocer su país } \\
\text { y sus costumbres personalmente" }\end{array}$ \\
\hline $\begin{array}{l}\text { Esp } \\
\text { en } \mathrm{E}\end{array}$ & $\begin{array}{l}\text { "Desaceleración del ritmo de clase, ya que algunos Erasmus no se } \\
\text { enteran" } \\
\text { "Cuando hay un número alto de alumnos Erasmus, hay poca relación } \\
\text { con los alumnos españoles" } \\
\text { "Poca participación en trabajos de grupo" } \\
\text { "Los españoles se relacionan poco con los Erasmus por falta de } \\
\text { comunicación, ya que el nivel de idiomas en España es bajo" }\end{array}$ \\
\hline $\mathrm{Bi}$ & "Ver las opiniones y el modo de pensar de otras personas de diferentes \\
\hline
\end{tabular}




\begin{tabular}{|c|c|}
\hline $\begin{array}{l}\text { Tipo de } \\
\text { estudiante }\end{array}$ & Comentario \\
\hline (Erasmus) & $\begin{array}{l}\text { nacionalidades" } \\
\text { "Aprovechar la oportunidad de oír la lengua" } \\
\text { "Es difícil acostumbrarse a la manera de interactuar entre los alumnos y } \\
\text { los profesores y hablar de tú con los profesores" }\end{array}$ \\
\hline $\begin{array}{l}\text { Española } \\
\text { en España }\end{array}$ & $\begin{array}{l}\text { "Poder experimentar con ellos y ayudarles a aprender nuestro idioma" } \\
\text { "Cuando se van es difícil olvidarlos" } \\
\text { "Puedes crear relaciones muy buenas para el futuro" } \\
\text { "Me encanta relacionarme con extranjeros y ayudarlos a su adaptación" }\end{array}$ \\
\hline Español & $\begin{array}{l}\text { "Es una satisfacción poder enseñar de una manera más personal } \\
\text { nuestro idioma" }\end{array}$ \\
\hline $\begin{array}{l}\text { Español } \\
\text { en España }\end{array}$ & $\begin{array}{l}\text { "Puede darse el caso de que los Erasmus se junten sólo entre ellos y no } \\
\text { se relacionen mucho con los españoles" } \\
\text { "Puede que algunos alumnos competitivos vean que a los Erasmus se } \\
\text { les dan facilidades y ayudas con respecto a la asignatura" } \\
\text { "Pienso que en un futuro esto nos servirá para poder relacionarnos y } \\
\text { mantener buenas relaciones en el trabajo con las diferentes personas } \\
\text { que compartamos trabajo" }\end{array}$ \\
\hline $\begin{array}{l}\text { Español } \\
\text { en España }\end{array}$ & $\begin{array}{l}\text { "A veces es difícil la comunicación entre españoles y Erasmus, aunque } \\
\text { siempre se establecen relaciones buenas" }\end{array}$ \\
\hline $\begin{array}{l}\text { Español } \\
\text { en España }\end{array}$ & "Sirve para tener nuevas amistades" "Se juntan los Erasmus entre ellos" \\
\hline $\begin{array}{l}\text { Español } \\
\text { en España }\end{array}$ & $\begin{array}{l}\text { "Poder conocer compañeros que me enseñen a enfocar un problema } \\
\text { rutinario desde otra perspectiva, así como poder ver cómo trabajan a } \\
\text { través de sus exposiciones" } \\
\text { "El idioma nunca fue un hándicap"'Ellos le han dado frescura al grupo" }\end{array}$ \\
\hline
\end{tabular}

Como se desprende de los comentarios de los estudiantes, en general todos valoran muy positivamente el poder practicar y mejorar el nivel de un idioma extranjero. Además, casi todos los entrevistados coinciden en las posibilidades de conocer otra 
cultura y otra forma de pensar y hacer las cosas diferentes a la propia, así como en las posibilidades de visitar otros países.

En cuanto al trabajo en grupo, cabe destacar que en el caso de que el profesor no obligue a los alumnos a formar grupo con los españoles la tendencia general es a que los Erasmus, aun siendo de nacionalidades diferentes se junten entre ellos, lo que posibilita las mismas competencias mencionadas, perfeccionamiento de idioma extranjero, conocimiento de otra cultura, etc.

Sin embargo, también hay opiniones desfavorables, como las que apuntan a que los estudiantes extranjeros pueden ralentizar el ritmo de la clase, puesto que algunos no conocen bien el idioma y que los estudiantes extranjeros, tanto los que vienen a España como los españoles que van a otros países se sienten en desventaja puesto que el sistema de trabajo, los casos prácticos, etc. se enmarcan en un entorno cultural diferente al suyo propio, lo que dificulta su aprendizaje.

\section{DISCUSIóN}

Las aulas universitarias europeas están actualmente configuradas por alumnos de diversas nacionalidades, lo que garantiza sin ninguna duda la multiculturalidad existente. Esto es así, en gran parte por las facilidades del ya consolidado programa Erasmus, que viene desplazando alumnos de unos países a otros desde hace más de 20 años. Recientemente, también se vienen incorporando a las universidades españolas estudiantes iberoamericanos, gracias a convenios con diversos países. Toda esta multiculturalidad enriquece tanto a alumnos como a profesores, puesto que podemos ver otras formas de pensar, otras formas de actuar, y en definitiva otras perspectivas de enfocar la realidad. Este bagaje cultural debe dar un paso hacia delante, consiguiendo dar el salto desde la multiculturalidad hacia la interculturalidad. Sin embargo, para que exista un enriquecimiento mutuo, debe también existir un intercambio entre las diferentes culturas que existen en un mismo espacio físico. Así, no es suficiente, que en el aula convivan estudiantes de varias nacionalidades (sentados y mirando al profesor, sin interactuar entre ellos), sino que debemos conseguir que se produzca una comunicación comprensiva entre ellos, lo que a su vez proporciona el reconocimiento y la valoración (intrínseca e extrínseca) de cada una de las culturas en un marco de igualdad.

El trabajo en grupos formados por alumnos de varios países tiene tanto ventajas como inconvenientes.

Respecto a las ventajas:

1. Trabajar con estudiantes de diferentes nacionalidades puede ayudarnos a mejorar las habilidades sociales de los alumnos.

2. Además, las aptitudes de trabajo en equipo de los componentes del grupo de trabajo tendrán que ser aún mayores al trabajar con estudiantes extranjeros, para poder llegar al entendimiento y a unos buenos resultados.

3. Los estudiantes se enriquecerán de los distintos estilos de vida y las distintas culturas.

4. Y puede ayudar al refuerzo o aprendizaje de nuevas lenguas de todos los estudiantes.

Respecto a los inconvenientes que podemos encontrar:

1. Puede que las diferencias en cuanto a las culturas, los horarios, el idioma, entre otros, compliquen el trabajo en grupo.

2. Podrían darse malentendidos dadas las diferentes culturas. 
3. Y también pueden darse entre los estudiantes prejuicios y establecimiento de estereotipos debido al desconocimiento de culturas diferentes a la propia, teniendo imágenes preconcebidas.

4. En aquellos casos en los que el profesor no obliga a integrar en cada grupo de españoles a un estudiante Erasmus, la tendencia general es que los Erasmus se junten entre ellos, lo que no favorece la interculturalidad entre españoles y extranjeros, pero sí entre los extranjeros que suelen formar grupos con otros extranjeros de diferentes nacionalidades.

Todos estos inconvenientes pueden llevar a 3 situaciones o problemas intragrupo:

1. Que una de las culturas sea la dominante y el resto del grupo se escaquee (efecto parásito).

2. Que haya una disgregación intragrupo, es decir, que un mismo grupo de trabajo se separe en varios, y que aunque todos trabajen, no lo hagan de forma cooperativa. $Y$ por último:

3. Que algún miembro del grupo se sienta fuera de lugar y acabe por abandonar.

En conclusión y resumiendo las distintas opiniones y experiencias vemos que destacan las ventajas del trabajo cooperativo con estudiantes de diferentes nacionalidades. Este tipo de trabajo en un entorno multicultural tendrá efectos positivos sobre el desarrollo personal de los diferentes estudiantes, ya que llevará al enriquecimiento tanto en la forma de trabajo como en el conocimiento de distintos modos de vida.

La interculturalidad puede ganar la batalla a la multiculturalidad en el aula, con la ayuda del profesor y la involucración de los estudiantes nativos y extranjeros y las ventajas pueden ser mayores que los inconvenientes para así conseguir un desarrollo personal más profundo.

\section{REFERENCIAS}

Amani, C. (2004). La escuela intercultural: regulación de conflictos en contextos multiculturales. J. G. Lara (Ed.). Los Libros de la Catarata.

Buendía, L. (1996). "Formación de los profesores para una escuela intercultural". En I Jornadas sobre invernaderos e inmigrantes: Marginación y educación intercultural. Almería.

De-Juan-Vigaray, M. D. (2007) Methodology for teaching Erasmus students: An experience teaching "Merchandising and sales promotions", Proceedings del International Technology, Education and Development Conference. IATED, Valencia (Spain). 07-09 marzo 2007.

De-Juan-Vigaray, M. D. y Barra, P. (2012) 243267 Los programas internacionales con docencia en inglés en Economía: el reto de integrar al estudiante español. $X$ Jornadas de Redes de Investigación en Docencia Universitaria Universidad de Alicante, organizadas por el Vicerrectorado de Planificación Estratégica Instituto de Ciencias de la Educación, 7 y 8 junio, Alicante (España). 
http://web.ua.es/es/ice/jornadas-redes/ Investigación enmarcada en las actividades de la RED y GITE IDOi de la UA.

De Miguel, M. (Coord.) (2006a). Metodologías de enseñanza y aprendizaje para el desarrollo de competencias. Orientaciones para el profesorado universitario ante el Espacio Europeo de Educación Superior. Madrid: Alianza.

De Miguel, M. (Dir.) (2006b). Modalidades de enseñanza centradas en el desarrollo de competencias. Orientaciones para promover el cambio metodológico en el Espacio Europeo de Educación Superior. Oviedo: Universidad de Oviedo.

Del Arco Bravo, I. (1998). Hacia una escuela intercultural. El profesorado: formación y expectativas. Lérida: EdicionsUniversitat de Lleida.

Delors, J. (coord.) (1996): Informe Unesco. La educación encierra un tesoro. Madrid, Santillana.

García Parejo, I., Pérez Milans, M. y Patiño Santos, A. (2008). La educación intercultural en las aulas multiculturales de la comunidad de Madrid: una brecha entre la teoría y la práctica. En Segundas Lenguas e Inmigración en red, 1 pp. 6071.

Jordán, J. A. (1996). Propuestas de Educación Intercultural para profesores. Barcelona: CEAC.

Jordán, J. A. (1997). Propuestas de educación intercultural para profesores. Barcelona, Ceac.

López López, M. C. y Hinojosa Pareja, E. F. (2010). Hacia una educación intercultural: estado de la investigación en el contexto nacional. En: E. Soriano, A. González y R.M. Zapata (eds.), Retos Internacionales ante la Interculturalidad. Almería: Editorial Universidad de Almería, 2010. pp. 494-503.

Merino, J. y Muñoz, A. (1995). Ejes de debate y propuestas de acción para una pedagogía intercultural. Revista de Educación, 307, pp. 127-162.

Quintana, J. M. (1992)."Características de la educación multicultural". En A.A.V.V. Educación Multicultural e intercultural. Granada: Impredisur.

Parra, M.C. y Peña, B. (2012). El aprendizaje cooperativo mediante actividades participativas. Anales de la Universidad Metropolitana, 12 (2), 15-37.

Sáez Alonso, Rafael (2006). La educación intercultural. Revista de educación (M.E.C.), 339 , pp. 859-881. 
Sedano, A. M. (2001). Enfoques y modelos de educación multicultural e intercultural. Education (Universidad Complutense y Universidad de Manitoba-Canadá), 1, 81106.

Traver Martí, J. A. y García López, R. (2007). Construcción de un cuestionario-escala sobre actitud del profesorado frente a la innovación educativa mediante técnicas de trabajo cooperativo (CAPIC). Revista electrónica de Investigación Educativa, 9(1).

Trujillo, F. (2002). Aprendizaje cooperativo para la enseñanza de la lengua". Publicaciones de la Facultad de Educación y Humanidades- Campus de Melilla, 32, 147-162. Disponible en: http://meteco.ugr.es/lecturas/cooperacion.pdf [Consulta 2013, 5 de diciembre].

Wiersma, W. y Jurs, S. G. (2005). Research methods in Education. Octavaedición. Ed. Pearson.7

http://www.ine.es/ss/Satellite?L=es ES\&c=INECifrasINE C\&cid=1259940012843\&p= 1254735116567 \&pagename $=$ ProductosYServicios\%2FPYSLayout

http://www.mecd.gob.es/dms/mecd/educacion-mecd/areaseducacion/universidades/estadisticas-informes/estadisticas-informesdocumentum/datos-cifras/2012-2013-datos-y-cifras-sistema-universitarioespanol.pdf pág. 30/36

\section{AUTOR/ES:}

\section{María D. De-Juan-Vigaray}

Doctora en CC.EE por la Universidad Miguel Hernández y Profesora Titular de Comercialización e Investigación de Mercados de la Universidad de Alicante (UA). Imparte diversos cursos en Universidades europeas y americanas. Colaboradora como docente en la University of Florida (EE.UU) y en el Southampton Institute (Inglaterra), así como en Masters y Escuelas de Negocios españolas (ESADE, ESIC). Comparte su docencia entre UA y el IESEG de la Université de Lille (Lille y París). Autora de varios libros de marketing, entre ellos 'Comercialización y retailing' y 'Valores de los consumidores'; así como de artículos sobre retailing. Labor investigadora enfocada en el comportamiento de ir de compras, el retailing, y la investigación en docencia, campos donde ha publicado diversos artículos de impacto.

\section{María Concepción Parra Meroño}

Doctora en Administración y Dirección de Empresas. Licenciada en Ciencias Económicas y Empresariales. Autora de varios manuales universitarios sobre Marketing y Comportamiento del Consumidor. Ha publicado diversos artículos en revistas de impacto JCR relacionados con marketing, comunicación, comportamiento del consumidor e investigación educativa. Ha participado en números congresos nacionales e internacionales de reconocido prestigio. Profesora del área de Comercialización e investigación de Mercados en la Universidad Católica San Antonio de Murcia desde 
hace 15 años, donde imparte asignaturas del área en Grado y Postgrado. Cuenta con experiencia profesional en el sector público y privado.

\section{Miguel Ángel Beltrán Bueno}

Licenciado en Ciencias Económicas y Empresariales. Autor de varios manuales universitarios sobre Marketing y Comportamiento del Consumidor. Ha publicado diversos artículos en revistas de impacto JCR relacionados con marketing, comunicación, comportamiento del consumidor e investigación educativa. Ha participado en números congresos nacionales e internacionales de reconocido prestigio. Profesor del área de Comercialización e investigación de Mercados en la Universidad Católica San Antonio de Murcia desde hace 15 años, donde imparte asignaturas del área en Grado y Postgrado. Cuenta con más de 20 años de experiencia profesional en el sector privado. 